\title{
Layering, freezing, and re-entrant melting of hard spheres in soft confinement
}

\author{
Tine Curk, ${ }^{1,2}$ Anouk de Hoogh,${ }^{3}$ Francisco J. Martinez-Veracoechea, ${ }^{2}$ Erika Eiser, ${ }^{4}$ Daan Frenkel, ${ }^{2}$ \\ Jure Dobnikar, ${ }^{2,5, *}$ and Mirjam E. Leunissen ${ }^{3, \dagger}$ \\ ${ }^{1}$ Faculty of Natural Sciences and Mathematics, Koroška c. 160, SI-2000 Maribor, Slovenia \\ ${ }^{2}$ University of Cambridge, Lensfield Road, Cambridge CB2 IEW, United Kingdom \\ ${ }^{3}$ FOM Institute AMOLF, Science Park 104, 1098 XG, Amsterdam, The Netherlands \\ ${ }^{4}$ University of Cambridge, Cavendish Laboratory, J.J. Thomson Avenue, Cambridge CB3 OHE, United Kingdom \\ ${ }^{5}$ Institute Jožef Stefan, Jamova 39, SI-1000, Ljubljana, Slovenia
}

(Received 5 December 2011; published 21 February 2012)

\begin{abstract}
Confinement can have a dramatic effect on the behavior of all sorts of particulate systems, and it therefore is an important phenomenon in many different areas of physics and technology. Here, we investigate the role played by the softness of the confining potential. Using grand canonical Monte Carlo simulations, we determine the phase diagram of three-dimensional hard spheres that in one dimension are constrained to a plane by a harmonic potential. The phase behavior depends strongly on the density and on the stiffness of the harmonic confinement. While we find the familiar sequence of confined hexagonal and square-symmetric packings, we do not observe any of the usual intervening ordered phases. Instead, the system phase separates under strong confinement, or forms a layered re-entrant liquid phase under weaker confinement. It is plausible that this behavior is due to the larger positional freedom in a soft confining potential and to the contribution that the confinement energy makes to the total free energy. The fact that specific structures can be induced or suppressed by simply changing the confinement conditions (e.g., in a dielectrophoretic trap) is important for applications that involve self-assembled structures of colloidal particles.
\end{abstract}

DOI: 10.1103/PhysRevE.85.021502

PACS number(s): 64.75.-g, 68.65.Ac, 64.70.-p, 82.70.-y

\section{INTRODUCTION}

The behavior of particles in confined geometries is important in many different areas of physics and technology. This includes the physics of ions in electromagnetic traps [1], of dusty plasmas confined by external fields [2,3], of classical electrons in quantum wells [4], and of colloidal suspensions in narrow slits [5], as well as application-oriented topics in nanotechnology, (bio)lubrication, and the self-assembly of microstructured materials (e.g., Refs. [6-10]). It is well known that confinement effects can dramatically change the behavior of such systems, both quantitatively and qualitatively. For instance, when a suspension of colloidal hard spheres is confined in a wedge-shaped geometry, one observes a rich cascade of different equilibrium crystal structures as the wall spacing increases [11-14]. Such behavior contrasts sharply with the bulk phase diagram, which consists of a single, density-dependent liquid to face-centered-cubic crystal transition. The past few decades have seen many studies of the behavior of hard spheres between impenetrable walls (e.g., Refs. [13-16]), of charged particles under strong confinement (a model for trapped Coulombic or Yukawa particles, e.g., Refs. $[2,3,17,18]$ ) and of confined dipolar colloids (e.g., Refs. [19-21]). However, to the best of our knowledge, there have been no studies that systematically investigate the role played by the softness of the confining potential. This is an important issue to address, as more and more systems become available that involve some form of soft confinement. In the areas of colloid science and nanotechnology one can, for instance, think of suspensions confined in dielectrophoretic [22-24] or laser-optical fields [25,26], nanometric objects

\footnotetext{
*jd489@cam.ac.uk

${ }^{\dagger}$ m.e.leunissen@amolf.nl; www.amolf.nl
}

in charged slits [6], particles interacting with soft polymer substrates [27,28], or particles trapped at liquid-liquid or liquid-gas interfaces [29-33]. Using Monte Carlo simulations, we here study three-dimensional systems of hard spheres that in one dimension are constrained to a plane by a harmonic potential. Unlike hard boundaries, this soft potential well does not prescribe a particular confinement "width," can be continuously tuned from very strong to very weak confinement, and makes an energy contribution to the total free energy which depends on the exact particle positions. We highlight the unique properties imparted by such soft confinement by comparing the observed phase behavior with that of hard spheres between two hard walls [14], as well as with the behavior of a more complex system of highly charged particles and their counterions between neutral walls [34]. In the latter system, the particles experience a combination of an effective harmonic potential due to the counterions and long-range repulsive Coulomb interactions.

\section{MODEL}

We performed grand canonical Monte Carlo simulations of hard spheres that are constrained to a plane by a harmonic potential. Hard spheres do not interact with each other unless their cores overlap, and for any pair of particles the interaction potential is given by

$$
\frac{U_{\text {sphere-sphere }}}{k_{B} T}(r)= \begin{cases}0, & r \geqslant \sigma \\ \infty, & r<\sigma,\end{cases}
$$

where $k_{B}$ is the Boltzmann constant, $T$ is the absolute temperature, $r$ is the center-to-center distance of the particles, and $\sigma$ is the particle diameter, which we took as the unit of length in our simulations. We used periodic boundary conditions in the $x y$ plane (box size $L_{x} \times L_{y}=20 \times 20$, unless stated 
otherwise) and a soft confining harmonic potential centered around $z=0$, which acts on each particle individually and whose softness was set through the spring constant $k$ (in units of $\sigma^{-2}$ ):

$$
\frac{U_{\text {conf }}}{k_{B} T}(z)=\frac{k z^{2}}{2} .
$$

We performed simulations for different chemical potentials of the reservoir, starting with an empty box. Using cell lists and an early rejection scheme [35], we typically performed $6 \times 10^{10}$ Monte Carlo moves in which we attempted to insert, delete, or displace $\left(d_{\max }=0.05\right)$ a randomly chosen particle, where the fraction of insertion and deletion moves was fixed at 0.2 . We also performed simulations with an additional move in which the $L_{x} / L_{y}$ aspect ratio of the box was allowed to change while keeping the area $A=L_{x} L_{y}$ constant. In principle, this should prevent the box shape from dictating the structure of the particle packing, but in all cases the resulting structures were identical to those observed in a square box.

After thorough equilibration, we determined the number of particles $N$ in the simulation box and from this the density, $\rho=N / A$, and characterized the structure of the typically layered particle packings by calculating the fourfold $\left(q_{4}\right)$ and sixfold $\left(q_{6}\right)$ symmetric two-dimensional bond-order parameters in each of the layers [36]. The bond-order parameters consider all of the nearest neighbors of a given particle that lie approximately in the same $z$ plane. Based on geometric arguments and the observed particle distributions along the $z$ axis, the nearest neighbors were here defined as those particles residing within a center-to-center distance $r_{\mathrm{NN}} \leqslant 1.3$ of the particle of interest and with a height difference $\delta z_{\mathrm{NN}} \leqslant 0.3$. This definition excludes second-nearest neighbors, which in a close-packed square-symmetric layer reside at $r=\sqrt{2}$, and is also found to reliably discriminate between thermally broadened and adjacent layers along $z$. The ratio between the two bond-order parameters allowed us to distinguish between the three main types of structures found in our simulations: disordered liquid for $1 / 3 \leqslant q_{4} / q_{6} \leqslant 3$, hexagonally $(\triangle)$ packed for $q_{4} / q_{6}<1 / 3$, and square $(\square)$ packed for $q_{4} / q_{6}>3$. The crossover values were determined from plots of $q_{4} / q_{6}$ across the entire density range studied in the simulations, as well as the density probability distributions, which revealed the first-order phase transitions. We find that varying $r_{\mathrm{NN}}$ and $\delta z_{\mathrm{NN}}$ by \pm 0.1 does not affect the resulting phase diagram.

\section{RESULTS}

The phase diagram in Fig. 1 and the simulation snapshots in Fig. 2 provide an overview of the observed particle packings as a function of the density and spring constant. At high spring constants $(k \gtrsim 150)$, i.e., relatively strong confinement, and low densities the particles form a layer of disordered liquid $(L)$ at the minimum of the confining harmonic potential. At higher densities, the liquid freezes into one or more crystalline layers which have a hexagonal $(\triangle)$ or square $(\square)$ symmetry (Fig. 2), following an alternating sequence as the density increases: $1 \triangle \rightarrow 2 \square \rightarrow 2 \triangle \rightarrow 3 \square \rightarrow 3 \triangle \rightarrow \cdots$ (the integers indicate the number of particle layers that are formed). To rationalize these observations, we consider the limit $k \rightarrow \infty$ (or $T \rightarrow 0$ ) in which the entropic contribution to the free energy can

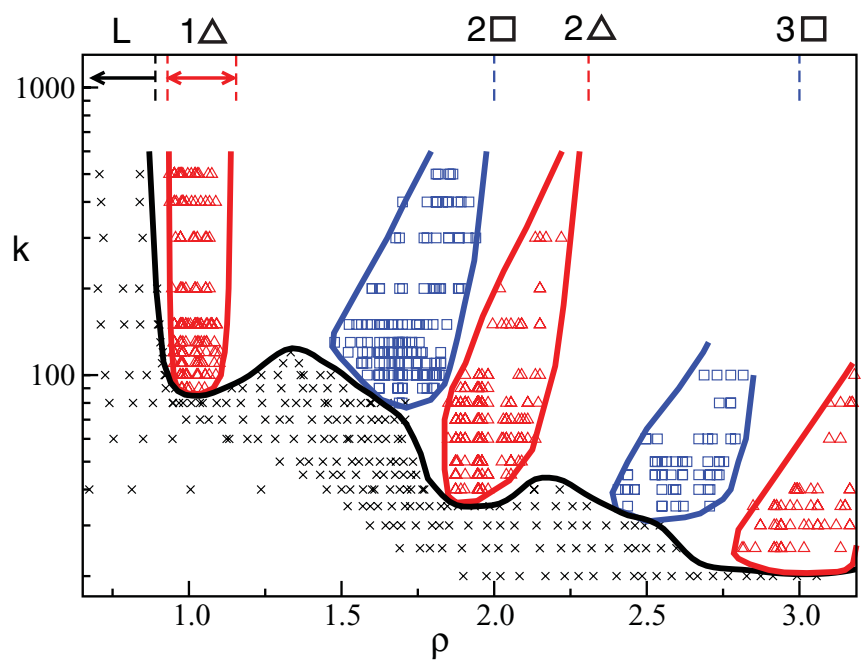

FIG. 1. (Color online) Phase diagram in the spring constantdensity representation. Every point represents the result of a single simulation run. Black crosses $(\times)$ : liquid. Red triangles $(\triangle)$ : hexagonally packed. Blue squares $(\square)$ : square packed (structures shown in Fig. 2). Approximate phase boundaries are indicated with solid lines. Dashed lines indicate the stable phases in the limit $k \rightarrow \infty$ (from Fig. 3).

be neglected and the energy contribution of the confining potential dominates: $F \approx E=\frac{k}{2} \sum z^{2}$, where the sum runs over all the particles in the system. Simple geometry then gives the energies of the perfect hexagonal and square packed structures, which scale linearly with the density because of the quadratic form of the confining potential (Fig. 3). The maximum density of each of the phases corresponds to close packing, e.g., $\rho_{\max }=2$ for $2 \square$ and $\rho_{\max }=4 / \sqrt{3}$ for $2 \triangle$. Starting at low density, the $L \rightarrow 1 \triangle$ transition is given by the two-dimensional hard disk freezing transition $[37,38]$, and the $1 \triangle$ phase (strongly constrained to $z=0$ and with negligible energy) is stable until close packing at $\rho=2 / \sqrt{3}$. Instead of continuously transforming into a $3 \triangle$ structure, the system then phase separates into $1 \triangle$ and $2 \square$. This phase separation can be understood in terms of a free energy minimization criterion, equivalent to the double-tangent construction. A similar argument applies to the subsequent transitions $2 \square \rightarrow$ $2 \triangle, 2 \triangle \rightarrow 3 \square$, and so on. At around seven layers the squaresymmetric structures eventually disappear in favor of the denser hexagonal packings, which have a face-centered cubic (fcc), hexagonal close packed (hcp), or a random hexagonal close packed (rhcp) structure, similar to the crystalline bulk phases of hard spheres.

The sequence of alternating hexagonal and square packings under strong harmonic confinement corresponds to the simple Pieranski picture of hard spheres confined between two hard walls [11]. However, we do not see any of the buckled, rhombic, and prism phases that are found to interpolate between these packings under hard-confinement conditions, when entropy solely determines the phase behavior [12-14]. By contrast, at high spring constants the behavior of the harmonically confined system is energy-dominated and the usual intervening phases are found to be unstable, because the second derivative of the (free) energy with respect to the 


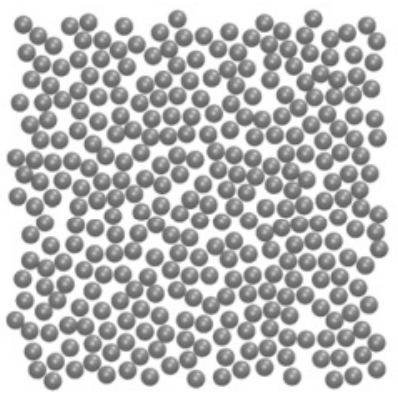

L; $\rho=0.8$

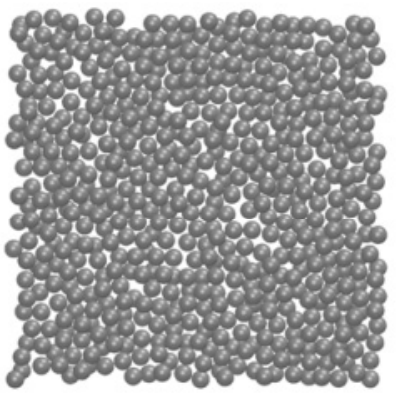

$\mathrm{L} ; \rho=1.39$
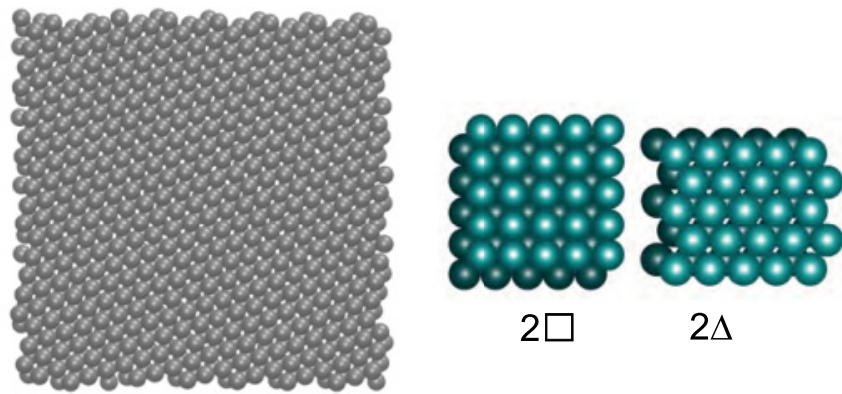

$2 \square$

$2 \Delta ; \rho=2.14$

FIG. 2. (Color online) Simulation snapshots at different densities (for $k=100$ ) and "ideal" schematic representations (lower right) of the spatial arrangement of the particles in the square ( $\square$ ) and hexagonally $(\triangle)$ packed structures.

density is negative. Furthermore, the intervening phases also appear to be unstable at finite spring constants, as we always observed a spontaneous melting (low spring constants) or phase separation (higher spring constants) of the system when it was initially prepared in one of these phases. In the absence of any stable intervening phases at higher spring constants, the coexistence regions between the stable hexagonal and square packed phases-which can only exist with low free energy at certain densities due to the integer number of layers-are wider than under hard-confinement conditions. (Note, by the way, that in order to approach the hard-confinement limit one would need to increase the exponent of the confining potential rather than the prefactor.) We point out that the alternating hexagonal and square-symmetric packings appear to be a common property of different types of confined repulsive particle systems $[5,14,34,39]$, while the character of the intervening phases seems to depend more strongly on the exact details of the particle-particle interactions and the confining potential. For example, at high spring constants

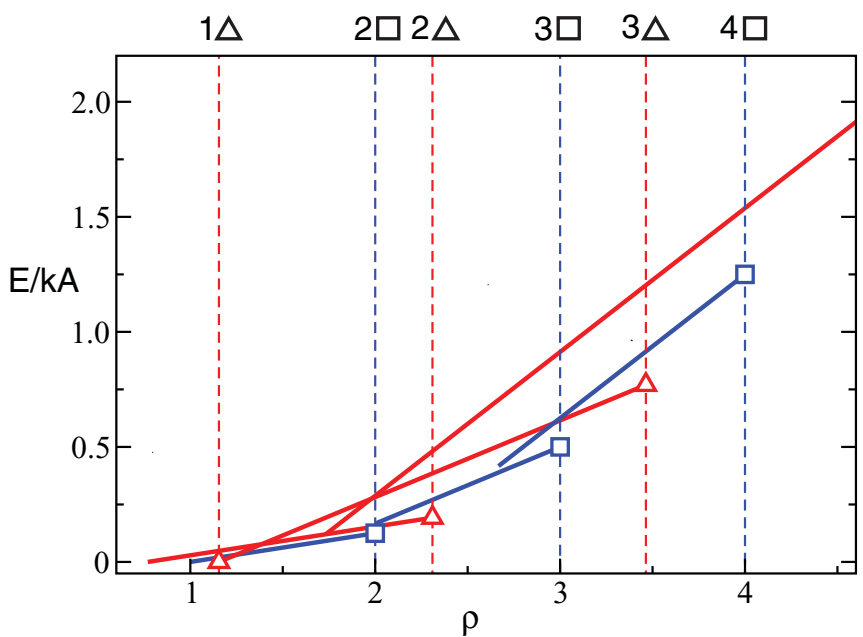

FIG. 3. (Color online) Normalized energies per unit area of the hexagonal (red lines, $\triangle$ ) and square (blue lines, $\square$ ) packed structures in the limit $k \rightarrow \infty$.

we do not observe any new phases between $1 \triangle$ and $2 \square$ in the harmonic potential, while the same hard spheres in a hard slit would form an intermediate buckled bilayer structure $(2 \mathcal{B})$ [12-14], and while charged particles in an effective harmonic potential are expected to form the sequence $1 \triangle \rightarrow 3 \triangle \rightarrow 2 \square$ in the limit $T \rightarrow 0$ [34]. In the latter system, which considered pointlike particles, the long-ranged repulsive Coulomb interactions between the particles compete with the attraction to the minimum of the confining potential.

Remarkably, as the harmonic confinement becomes softer (lower spring constant), we do find stable intervening phases, which, however, are not ordered. Instead of the more commonly observed solid-to-solid transformations, the system undergoes a couple of re-entrant melting transitions that give rise to intervening liquid phases, with triple points around $\{k=120, \rho=1.2\}$ and $\{k=45, \rho=2.25\}$. Thus at around $k \approx 100$ we observe freezing into a stable $1 \triangle$ phase, which then remelts into a disordered liquid before freezing again into the $2 \square$ phase (Fig. 2), while for higher densities we find the same alternating sequence of hexagonal and square packings as before. We further see that the softer the confinement, the higher the density at which the initial liquid phase still persists and at sufficiently low spring constants $(k \lesssim 80)$ the first stable ordered structures actually consist of more than one layer. For example, at $k \approx 40$ the first ordered phase is $2 \triangle$, followed by re-entrant melting, the $3 \square$ structure, and then the other multilayered ordered phases, whereas for $k \lesssim 35$ we only observe the latter, without any intermediate melting.

To make sure that the re-entrant liquid phases did not represent finite-size artifacts, we performed simulations with three different box sizes $\left(L_{x}=L_{y}=10,20\right.$, or 50) for $k=100$ (Fig. 4). It can be seen that the results for the different system sizes are essentially identical and that there is a clear transition from the $1 \triangle$ phase to a re-entrant liquid and then to the $2 \square$ phase, as reflected by the $q_{4} / q_{6}$ bond-order parameter ratio. We also determined the density probability distributions at coexistence [40,41] using histogram-reweighing [42] and expanded ensemble [43] simulations (insets in Fig. 4). For both the $1 \triangle \rightarrow L$ and the $L \rightarrow 2 \square$ transitions we find 


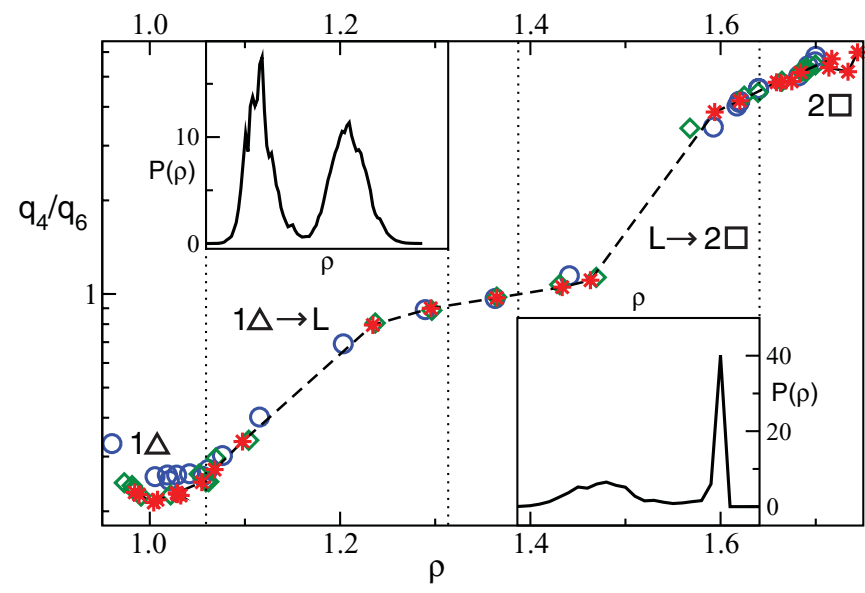

FIG. 4. (Color online) Density-dependent re-entrant melting between the $1 \triangle$ and $2 \square$ phases, for $k=100$ and different sizes of the simulation box $\left(L=L_{x}=L_{y}\right)$. Red stars: $L=50$. Green diamonds: $L=20$. Blue circles: $L=10$. The insets give the probability distribution of observing a given density at coexistence chemical potential on the same density scale as the main plot.

bimodal probability distributions that are characteristic of a first-order phase transition. In addition, Fig. 5 shows the spatial distribution of the particles in the soft confining potential for different densities outside the coexistence region. As expected, in the $1 \triangle$ phase the particle distribution has a single peak centered at the energy minimum $z=0$, and in the $2 \square$ phase there are two peaks which are symmetrically located with respect to $z=0$. The intervening re-entrant liquid starts out with a flat and broad distribution of the particles at low densities, which then continuously transforms into a doubly peaked profile as the density increases. Interestingly, this occurs without further ordering of the particles in the $x y$ plane so that the result is a layered liquid. Others have shown that if the same hard spheres are confined between impenetrable hard walls, a highly ordered buckled structure forms when the wall separation is larger than required for the $1 \triangle$ phase but too small

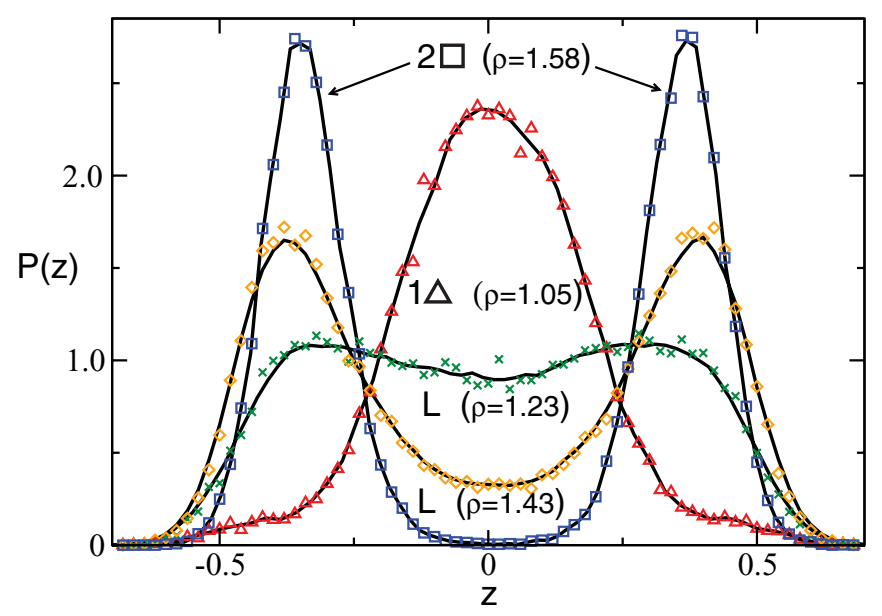

FIG. 5. (Color online) Particle distribution along the axis of confinement $(z)$ for $k=100$ and different densities. Red triangles: $1 \triangle, \rho=1.05$. Green crosses: liquid, $\rho=1.23$. Yellow diamonds: liquid, $\rho=1.43$. Blue squares: $2 \square, \rho=1.58$. for the $2 \square$ phase [12-14]. This buckled $2 \mathcal{B}$ phase optimizes the particle packing in the available space between the two walls by splitting the $1 \triangle$ structure into rows of particles that alternate in height, effectively forming a crystal of two interpenetrating rectangular layers, which continuously transforms into the $2 \square$ structure as the wall separation increases. In the harmonically constrained system, the re-entrant layered liquid fulfills a similar interpolating role between the one- and two-layer ordered phases, but the additional energy considerations and greater positional freedom under soft confinement, together with the absence of long-ranged particle interactions, favor disordered over ordered phases.

\section{CONCLUSIONS}

We have identified the stable phases of hard spheres under harmonic confinement as a function of the density and the softness of the confining potential. We find the well-known "base" sequence of alternating hexagonal and square-symmetric packings $(1 \triangle \rightarrow 2 \square \rightarrow 2 \triangle \rightarrow 3 \square \rightarrow$ $3 \triangle \rightarrow \cdots)$, which thus appears to be quite insensitive to the details of the (confining) interactions. When we look at the presence of stable intervening phases the soft-confined hard-sphere system is clearly different from hard-confined systems, though. Instead of the usual highly ordered interpolating particle packings, we observe phase separation between the hexagonally and square packed structures under strong confinement and disordered re-entrant liquid $(L)$ phases under weaker confinement. For $1 \triangle \rightarrow L$ and $L \rightarrow 2 \square$, we have shown that the re-entrant melting (freezing) transition has a first-order character and that the liquid develops a layered structure, without further ordering of the particles. We argue that the re-entrant and phase-separating behaviors are due to the fact that the penetrable soft harmonic potential on the one hand offers more positional freedom than hard-wall confinement does, while, on the other hand, making an important energy contribution to the overall free energy of the system which depends on the exact particle positions (in addition to the usual entropic considerations for hard spheres). The net result is a smaller diversity of crystalline packings as compared to hard spheres between two hard walls. We expect that other soft confining potentials may well have a similar effect, although the details of the phase diagram will likely be different. The fact that certain ordered structures can be reliably obtained, while many other structures can be induced or suppressed on demand through a variation of the confinement conditions is important for applications that involve self-assembled structures of colloidal particles. Interestingly, it should be possible to realize harmonic confining potentials with dynamically tunable softness experimentally, for instance, in a dielectrophoretic trap.

\section{ACKNOWLEDGMENTS}

This work was supported through the research program of the Foundation for Fundamental Research on Matter (FOM), which is part of the Netherlands Organization for Scientific Research (NWO), by the Slovenian Research Agency through Grant No. P1-0055, by the European Research Council through the Advanced Research Grant COLSTRUCTION (No. 
RG52356), and by the 7th Framework Program through the ITN network COMPLOIDS (No. RG234810). D.F. acknowledges support through a Wolfson Merit Award 2007/R3 of the Royal Society of London and an EPSRC Program Grant EP/I001352/1. T.C. acknowledges support from the Erasmus work placement scheme.
[1] J. Walz, I. Siemers, M. Schubert, W. Neuhauser, and R. Blatt, Europhys. Lett. 21, 183 (1993).

[2] H. Totsuji, T. Kishimoto, and C. Totsuji, Phys. Rev. Lett. 78, 3113 (1997).

[3] R. Messina and H. Löwen, Phys. Rev. Lett. 91, 146101 (2003).

[4] K. Esfarjani and Y. Kawazoe, J. Phys.: Condens. Matter 7, 7217 (1995).

[5] H. Löwen, J. Phys.: Condens. Matter 21, 474203 (2009).

[6] M. Krishnan, N. Mojarad, P. Kukura, and V. Sandoghdar, Nature (London) 467, 692 (2010).

[7] D. Leckband and J. Israelachvili, Q. Rev. Biophys. 34, 105 (2001).

[8] A. L. Copley (Ed.), Proceedings of the Fourth International Congress on Rheology (John Wiley \& Sons, Inc., New York, 1963).

[9] I. Cohen, T. G. Mason, and D. A. Weitz, Phys. Rev. Lett. 93, 046001 (2004).

[10] Y. A. Vlasov, X.-Z. Bo, J. C. Sturm, and D. J. Norris, Nature (London) 414, 289 (2001).

[11] P. Pieranski, L. Strzelecki, and B. Pansu, Phys. Rev. Lett. 50, 900 (1983).

[12] B. Pansu, Pi. Pieranski, and Pa. Pieranski, J. Phys. France 45, 331 (1984).

[13] S. Neser, C. Bechinger, P. Leiderer, and T. Palberg, Phys. Rev. Lett. 79, 2348 (1997).

[14] A. Fortini and M. Dijkstra, J. Phys.: Condens. Matter 18, L371 (2006).

[15] M. Schmidt and H. Löwen, Phys. Rev. Lett. 76, 4552 (1996).

[16] M. Schmidt and H. Löwen, Phys. Rev. E 55, 7228 (1997).

[17] D. H. E. Dubin, Phys. Rev. Lett. 71, 2753 (1993).

[18] A. B. Fontecha, H. J. Schöpe, H. König, T. Palberg, R. Messina, and H. Löwen, J. Phys.: Condens. Matter 17, S2779 (2005).

[19] K. Zahn, R. Lenke, and G. Maret, Phys. Rev. Lett. 82, 2721 (1999).

[20] N. Osterman, D. Babič, I. Poberaj, J. Dobnikar, and P. Ziherl, Phys. Rev. Lett. 99, 248301 (2007).

[21] J. Dobnikar, J. Fornleitner, and G. Kahl, J. Phys.: Condens. Matter 20, 494220 (2008).

[22] A. Docoslis and P. Alexandridis, Electrophoresis 23, 2174 (2002).

[23] M. T. Sullivan, K. Zhao, A. D. Hollingsworth, R. H. Austin, W. B. Russel, and P. M. Chaikin, Phys. Rev. Lett. 96, 015703 (2006).
[24] M. E. Leunissen and A. van Blaaderen, J. Chem. Phys. 128, 164509 (2008).

[25] A. Chowdhury, B. J. Ackerson, and N. A. Clark, Phys. Rev. Lett. 55, 833 (1985).

[26] M. C. Jenkins and S. U. Egelhaaf, J. Phys.: Condens. Matter 20, 404220 (2008).

[27] P. Akcora, H. Liu, S. K. Kumar, J. Moll, Y. Li, B. C. Benicewicz, L. S. Schadler, D. Acehan, A. Z. Panagiotopoulos, V. Pryamitsyn, V. Ganesan, J. Ilavsky, P. Thiyagarajan, R. H. Colby, and J. F. Douglas, Nature Mater. 8, 354 (2009).

[28] L. Di Michele, T. Yanagishima, A. R. Brewer, J. Kotar, E. Eiser, and S. Fraden, Phys. Rev. Lett. 107, 136101 (2011).

[29] R. Aveyard, B. Binks, and J. Clint, Adv. Colloid Interface Sci. 100-102, 503 (2003).

[30] E. M. Herzig, K. A. White, A. B. Schofield, W. C. K. Poon, and P. S. Clegg, Nature Mater. 6, 966 (2007).

[31] M. E. Leunissen, A. van Blaaderen, A. D. Hollingsworth, M. T. Sullivan, and P. M. Chaikin, Proc. Nat. Acad. Sci. USA 104, 2585 (2007).

[32] F. Bresme and M. Oettel, J. Phys.: Condens. Matter 19, 413101 (2007).

[33] L. Isa, E. Amstad, K. Schwenke, E. Del Gado, P. Ilg, M. Kröger, and E. Reimhult, Soft Matter 7, 7663 (2011).

[34] E. C. Oğuz, R. Messina, and H. Löwen, J. Phys.: Condens. Matter 21, 424110 (2009).

[35] D. Frenkel and B. Smit, Understanding Molecular Simulation (Academic Press, London, 2002).

[36] P. J. Steinhardt, D. R. Nelson, and M. Ronchetti, Phys. Rev. B 28, 784 (1983).

[37] E. Velasco and L. Mederos, Phys. Rev. B 56, 2432 (1997).

[38] K. Binder, S. Sengupta, and P. Nielaba, J. Phys.: Condens. Matter 14, 2323 (2002).

[39] M. Kahn, J.-J. Weis, and G. Kahl, J. Chem. Phys. 133, 224504 (2010).

[40] J. R. Errington and A. Z. Panagiotopoulos, J. Chem. Phys. 109, 1093 (1998).

[41] F. J. Martinez-Veracoechea, B. Bozorgui, and D. Frenkel, Soft Matter 6, 6136 (2010).

[42] A. M. Ferrenberg and R. H. Swendsen, Phys. Rev. Lett. 61, 2635 (1988).

[43] A. P. Lyubartsev, A. A. Martsinovski, S. V. Shevkunov, and P. N. Vorontsovvelyaminov, J. Chem. Phys. 96, 1776 (1992). 